\title{
El extracto etanólico de las flores de Laccopetalum giganteum (pacra-pacra) aumenta la fertilidad en ratas
}

\author{
Jorge Arroyo ${ }^{1,2}$, Alejandro Barreda ${ }^{3}$, Ernesto Ráez ${ }^{4}$, Bertha Jurado ${ }^{5}$, Gloria Moral ${ }^{5}$, \\ Jaime Martínez ${ }^{6}$, Christian Palomino ${ }^{6}$
}

Resumen

Palabras clave

\begin{abstract}
Objetivos: Determinar si la administración por vía oral del extracto etanólico de flores de Laccopetalum giganteum (Pacra-pacra) en ratas normales aumenta la fertilidad. Diseño: Estudio experimental. Lugar: Facultad de Medicina y Facultad de Farmacia y Bioquímica, Universidad Nacional Mayor de San Marcos. Material biológico: Ratas. Intervenciones: Cuarentiocho animales fueron divididos aleatoriamente en 2 grandes grupos, de 24 cada uno. El primero fue control, con solución salina fisiológica (SSF) $5 \mathrm{~mL} / \mathrm{kg}$ y el segundo con extracto vía oral, $300 \mathrm{mg} / \mathrm{kg}$, durante 15 días. Cada grupo consideró 6 hembras y 6 machos juntos, y 6 hembras y 6 machos separados. Los animales que estuvieron juntos fueron sacrificados, para observar la presencia de fetos en el útero; y a los animales que estuvieron separados, se les extrajo muestra de sangre, para conocer el nivel de testosterona en machos y LH, FSH, estrógenos y progesterona en hembras, expresándose en ug/dL; el dosaje hormonal se realizó por el método de electroquimioluminiscencia. Asimismo, se determinó GOT, GPT, urea y creatinina, según las técnicas utilizadas en el laboratorio clínico. Principales medidas de resultados: Gravidez, $F S H, L H$, estrógenos y progesterona en ratas hembras; testosterona en ratas machos. Resultados: Los flavonoides, compuestos fenólicos y taninos estuvieron en mayor cantidad en el extracto etanólico. El 100\% de ratas que recibió el extracto etanólico de la planta y estuvo junto a los machos resultó grávida; $y$, las separadas que recibieron el mismo extracto, presentaron incremento de FSH en las hembras y testosterona en los machos, comparativamente a los controles respectivos. Los niveles de GOT, GPT, urea y creatinina se encontraron dentro de los límites aceptados. Conclusiones: En condiciones experimentales, el extracto etanólico de las flores de Laccopetalum giganteum (pacra-pacra) incrementó la fertilidad en ratas normales.
\end{abstract}

Extractos vegetales; fertilidad; investigación.
Ethanolic flower extract from Laccopetalum giganteum (pacra-pacra) increases fertility in rats Abstract

Objective: To determine whether oral administration of the ethanolic flower extract from Laccopetalum giganteum (pacra-pacra) increases fertility in normal rats. Design:

1 Instituto de Investigaciones Clínicas, Facultad de Medicina Universidad Nacional Mayor de San Marcos. Lima, Perú.

2 Sección de Farmacología, Departamento Académico de Ciencias Dinámicas, Facultad de Medicina - Universidad Nacional Mayor de San Marcos. Lima, Perú.

3 Departamento Académico de Ginecología y Obstetricia, Facultad de Medicina - Universidad Nacional Mayor de San Marcos. Lima, Perú.

4 Instituto de Patología, Facultad de Medicina - Universidad Nacional Mayor de San Marcos. Lima, Perú.

5 Laboratorio de Farmacognosia y Medicina Tradicional. Facultad de Farmacia y Bioquímica - Universidad Nacional Mayor de San Marcos. Lima, Perú.

6 Post Grado - Universidad Nacional Mayor de San Marcos. Lima, Perú.
Experimental study. Setting: Faculty of Medicine and Faculty of Pharmacy and Biochemistry, Universidad Nacional Mayor de San Marcos. Biologic material: Rats. Interventions: Forty-eight rats were randomly distributed into 2 groups of 24 rats each. The first control group received solución salina fisiológica (SSF) $5 \mathrm{~mL} / \mathrm{kg}$ and the second group $300 \mathrm{mg} / \mathrm{kg}$ of the extract, orally, for 15 days. Each group had 6 female rats and 6 male rats living together and 6 female rats and 6 male rats lived separate. The animals living together were sacrificed in order to observe the fetuses in the uterus. The animals that lived separate had blood samples taken in order to determine testosterone levels in male rats and $\mathrm{LH}$, $\mathrm{FSH}$, estrogen and progesterone levels in female rats using electro chemiluminescence. At the same time, liver function tests, urea and creatinine tests were done. Main outcome measures: Pregnancy, FSH, LH, estrogens and progesterone in female rats; testosterone in male rats. 
Results: Flavonoids, tannins and phenolic compounds were found in higher quantities in the ethanolic extract. All female rats receiving the plant extract and living together with the male rats got pregnant and the ones that lived separate had increased levels of FSH. Male rats had increased levels of testosterone as compared to the control group. Liver function tests, urea and creatinine were within acceptable limits. Conclusions: The ethanolic flower extract from Laccopetalum giganteum (pacra-pacra) increased fertility in normal rats.

Key words: Plant extracts; fertility; research.

\section{INTRODUCCIÓN}

Aproximadamente entre 10 y $15 \%$ de las parejas sufren problemas de infertilidad. Con las actuales técnicas de reproducción asistida, muchas de ellas inician un programa de fecundación in vitro (FIV) y transferencia embrionaria (TE), con la esperanza de lograr una gestación. La tasa de gestación clínica por ciclo de FIV-inyección intracitoplasmática de espermatozoides (ICSI) es de alrededor de $30 \%$ $\left.{ }^{(}\right)$. La tasa de embarazo clínico medio por ciclo de FIV oscila entre $26 \%$ y $44,8 \%(2,3)$.

La salud humana es de gran importancia para el desarrollo y el progreso de un país. Se ha utilizado preparaciones o medicamentos herbarios desde épocas antiguas, en muchas partes del mundo, incluyendo la India. En años recientes, su uso como alternativa popular a la medicina moderna ha aumentado considerablemente en países desarrollados $\left({ }^{4-6}\right)$.

Diversos estudios demuestran actividad en la espermatogénesis y estimulación de la fertilidad de plantas altoandinas diversas $\left({ }^{7,8}\right)$. El Laccopetalum giganteum (pacra-pacra), planta nativa, extendida en la región altoandina, se le encuentra en las laderas calizas de la puna, entre 4200 a $4600 \mathrm{msnm}$, cuyas flores son utilizadas por los habitantes de la zona como estimulante de la actividad reproductiva en el hombre y sus animales.

El siguiente trabajo presenta la influencia del extracto etanólico de las flores de Laccopetalum giganteum (pacra-pacra) sobre la fertilidad en ratas, así como su seguridad a nivel hepático y renal.

\section{MÉTODOS}

Se utilizó 48 ratas albinas de cepa Holtzmann, con peso al inicio de la investigación de 180 a $200 \mathrm{~g}$, de 2 meses de edad, entre machos y hembras, distribuidos por igual cantidad, procedentes del Bioterio del Instituto Nacional de Salud del Ministerio de Salud, mantenidas en un ambiente a temperatura de $21^{\circ} \mathrm{C}$, con dieta y agua a libertad. Luego de un período de ambientación de 7 días y un día anterior al inicio del ensayo, fueron distribuidas al azar en 2 grandes grupos experimentales, de 24 ratas cada grupo; el grupo control recibió solución salina fisiológica (SSF) $5 \mathrm{~mL} / \mathrm{kg}$ y el grupo de estudio, el extracto etanólico de las flores de la planta Laccopetalum giganteum (pacra-pacra), en dosis de $300 \mathrm{mg} / \mathrm{kg}$. Ambos fueron administrados por vía oral durante 15 días. El primer grupo fue el grupo control, de 24 ratas, constituido por 6 machos y 6 hembras, juntos en una jaula, y 6 machos y hembras separados. El segundo grupo de 24 ratas estuvo conformado por 6 machos y 6 hembras juntos en la misma jaula, y 6 machos y 6 hembras separados.

Al finalizar el tratamiento, se obtuvo muestras de sangre para estudios bioquímicos y hormonales de las ratas que estuvieron en jaulas separadas; en tanto, las ratas que estuvieron juntas fueron sacrificadas para evaluar la fertilidad.

Las flores de la planta Laccopetalum giganteum (pacra-pacra) fueron recolectadas en Huaraz, departamento de Ancash. La identificación taxonómica de la planta fue realizada en el Museo de Historia Natural de la Universidad Nacional Mayor de San Marcos.

Para la preparación del extracto alcohólico, las flores frescas fueron lavadas y sometidas a deshidratación, a $40{ }^{\circ} \mathrm{C}$, por 48 horas; luego, fueron trituradas en un molino eléctrico, hasta obtener un polvo fino, macerándose con alcohol a $60^{\circ} \mathrm{C}$. Luego de los 15 días, se filtró y se llevó a estufa, a $40{ }^{\circ} \mathrm{C}$, en placas grandes, para la desecación respectiva, por 4 días, para llevarlo a extracto seco. Y al extracto seco se le realizó reacciones de identificación o coloración para determinar la presencia o ausencia de metabolitos secundarios haciendo uso de reactivos específicos, en los resultados se 
indicó presencia o ausencia del metabolito: $5 \mathrm{mg}$ de extracto problema con 5 gotas de reactivos $\left({ }^{9}\right)$.

El estudio farmacológico in vivo se realizó a las ratas hembras y machos que recibieron SSF y extracto etanólico de las flores de la planta Laccopetalum giganteum (pacra-pacra) y estuvieron en jaulas separadas; se les extrajo muestra de sangre, para dosaje de hormonas: testosterona en machos y en la hembras LH, FSH, estrógenos y progesterona, expresándose en $\mu \mathrm{g} / \mathrm{dL}$. El dosaje hormonal se hizo mediante el método electroquimioluminiscencia, que es una forma química seca, con una sensibilidad y especificidad de $99 \%$. Las ratas hembras que recibieron el extracto, así como las ratas hembras que recibieron suero fisiológico y estuvieron en jaulas junto a los machos, fueron sacrificados para retirarles el útero y evaluar la fecundidad o gravidez, por medio de la observación de los fetos presentes.

Se determinó los niveles de urea, creatinina y transaminasas, como indicadores de la función renal y hepática, mediante los métodos convencionales realizados en el Instituto de Investigaciones Clínicas de la Facultad de Medicina de la UNMSM.

Los datos obtenidos al evaluar el aumento de la actividad fertilizante fueron sometidos al análisis de varianza, y comparación de medias; para los diferentes análisis se consideró la $p<0,05$.

\section{RESULTADOS}

La clasificación taxonómica y descripción botánica de la especie se realizó en el Museo de Historia Natural de la Universidad Nacional Mayor de San Marcos (Constancia N.040-USM-2006). La muestra vegetal (planta completa) fue estudiada y clasificada como Laccopetalum giganteum (Wedd.) Ulbrich y tiene la siguiente posición taxonómica, según el sistema de Clasificación de Cronquist $\left({ }^{10}\right)$ :

División : magnoliophyta

Clase : magnoliopsida

Orden : ranunculales

Familia : ranunculaceae

Género : laccopetalum

Especie : Laccopetalum giganteum (Wedd.) Ulbrich Nombre vulgar: Pacra pacra
Se obtuvo un extracto etanólico con aspecto de masa homogénea, consistencia blanda, color verde petróleo, libre de partículas extrañas; el rendimiento fue de $9,4 \%$ de planta entera. El estudio fitoquímico demostró que los flavonoides, glicósidos, aminoácidos, esteroides y triterpenoides, compuestos fenólicos y taninos estuvieron en mayor cantidad en la fracción (Tabla 1).

En ratas hembras que recibieron la planta, se incrementó el nivel de FSH, en tanto que la $\mathrm{LH}$, el estradiol y la progesterona estuvieron reducidos $\mathrm{O}$ fue similar al normal sin planta (Figuras 1 y 2 ).

En los machos que recibieron la planta, se estimuló la producción de testosterona (Figura 3).

Los niveles de transaminasas (GOT, GPT), urea y creatinina se encontraron dentro de los límites permitidos para ratas, tanto en el grupo control como en el grupo de estudio (Figuras 4 y 5).

Las ratas hembras que estuvieron juntas a los machos y que recibieron la planta resultaron grávidas, en $100 \%$ de la muestra en estudio (Figuras 6 y 7).

\section{DISCUSIÓN}

El extracto alcohólico de las flores de Laccopetalum giganteum (pacra-pacra), al ser administrado por vía oral en dosis de $300 \mathrm{mg} / \mathrm{kg}$, a ratas normales hembras, aumentó los niveles de FSH, comparativamente al grupo control (Figura 1).

Tabla 1. Marcha fitoquímica del extracto de Laccopetalum giganteum (pacra pacra).

\begin{tabular}{llc}
\hline Reacción de: & Metabolito secundario & Extracto \\
\hline Gelatina & Taninos & ++ \\
Tricloruro férrico & Compuestos fenólicos & ++ \\
Dragendorff & Alcaloides & ++++ \\
Molisch & Azúcares & ++++ \\
NaOH 10\% & Quinonas & ++ \\
Vainillin sulfúrico & Glicósidos & ++++ \\
Liebermann & Esteroides y triterpenos & ++++ \\
Shinoda & Flavonoides & +++ \\
Ninhidrina & Aminoácidos libres & ++ \\
\hline
\end{tabular}

$(++++)$ muy abundante cantidad, $(+++)$ abundante cantidad, $(++)$ regular cantidad o positivo, $(+)$ poca cantidad o trazas. 


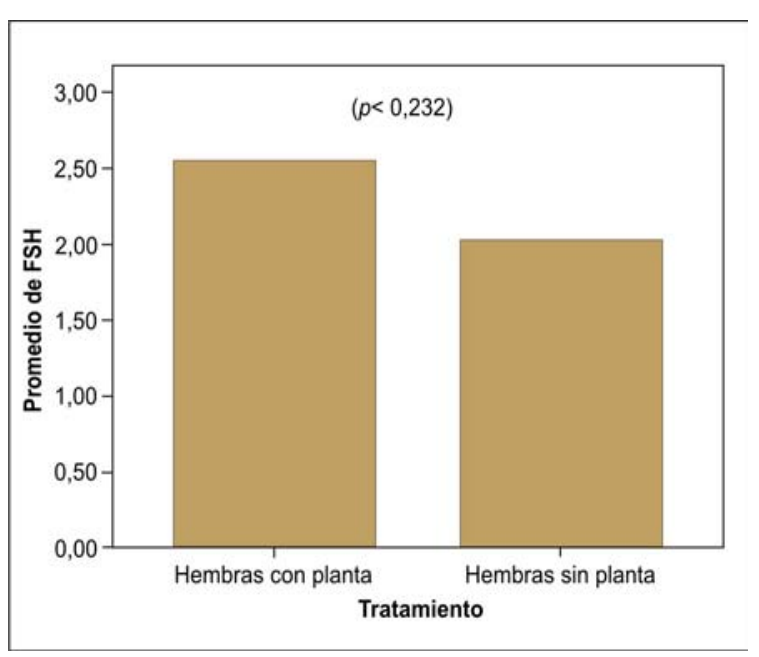

Figura 1. Nivel de la hormona foliulo-estimulante (FSH) en el extracto etanólico de las flores de Laccopetalum giganteum (pacra pacra), en ratas normales hembras.

La función reproductora en el hombre implica al sistema neuroendocrino y a las gónadas, que presentan dos funciones bien diferenciadas, espermatogénesis y esteroidogénesis. Ambas funciones son controladas por vía endocrina, desde la hipófisis anterior, que secreta dos gonadotropinas, la hormona folículo estimulante (FSH) y la hormona luteinizante $(\mathrm{LH})$. Sus funciones son: FSH actúa sobre las células de Sértoli, localizadas en el interior de los tubos seminíferos, estimulando la es-

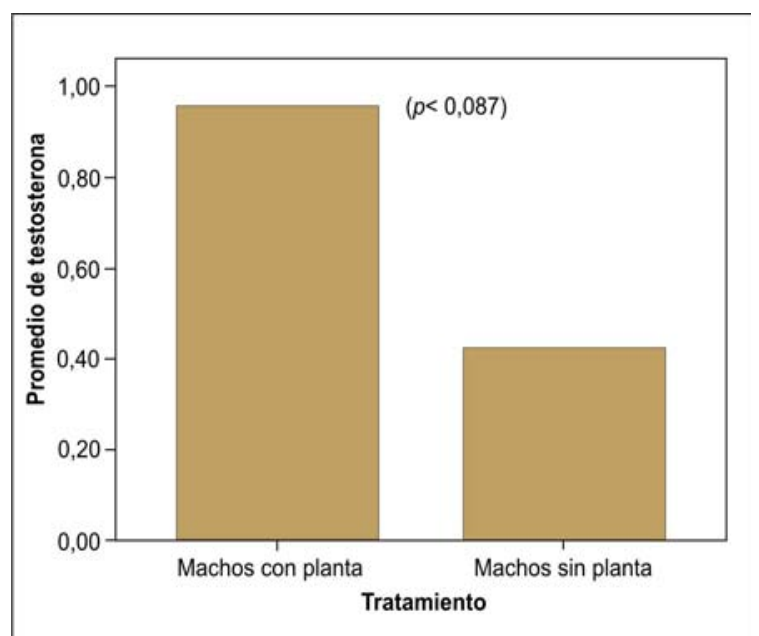

Figura 3. Extracto etanólico de las flores de Laccopetalum giganteum (pacra pacra), en ratas normales machos.

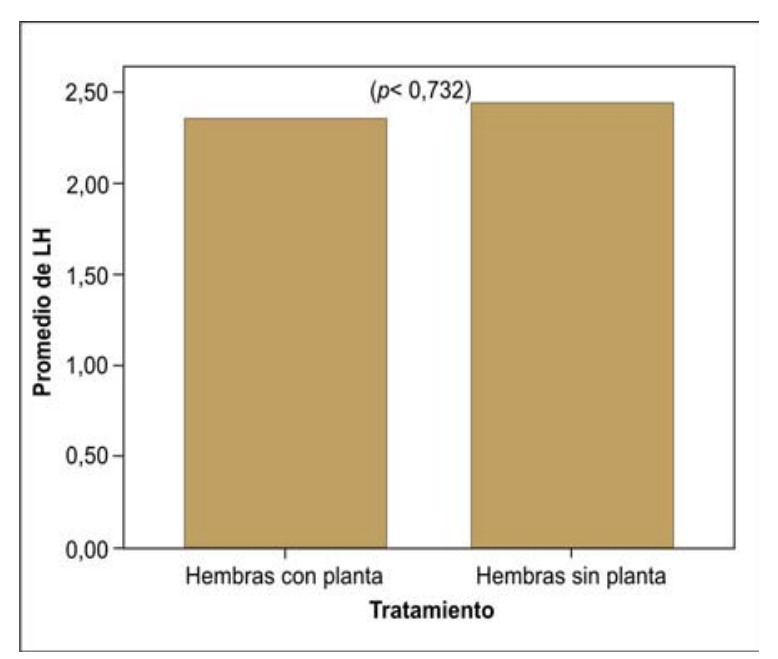

Figura 2. Nivel de la hormona luteinizante (LH) en el extracto etanólico de las flores de Laccopetalum giganteum (pacra pacra), en ratas normales hembras.

permatogénesis o producción de espermatozoides; LH actúa sobre las células de Leydig, en el tejido intersticial, provocando el proceso de esteroidogénesis o producción de hormonas masculinas (andrógenos), cuyo principal producto es la testosterona. La testosterona regula la secreción de $\mathrm{LH}$, actuando sobre el hipotálamo y la hipófisis anterior, en un proceso de homeostasis negativa $\left({ }^{11}\right)$.

Con los hallazgos descritos, se postula que el extracto etanólico de las flores de Laccopetalum giganteum (pacra-pacra) podría actuar directamente sobre el hipótalamo y la hipófisis, favoreciendo la liberación de los factores de la FSH, y sobre la hipófisis, permitiendo la producción de gonadotropina hipofisiaria FSH.

Por otro lado, al administrar el extracto alcohólico de las flores de Laccopetalum giganteum (pacra-pacra), en ratas normales machos estimula la producción de testosterona (Figura 3), posiblemente por metabolitos secundarios presentes en la planta (Tabla 1), que actuarían a nivel del hipotálamo liberando la neuregulina (Nrg). Este, a su vez, actuaría a nivel hipofisiario, liberando inicialmente LH y activando las células de Leydig $\left({ }^{12}\right)$. Posteriormente, la LH caería por retroalimentación negativa, debido al incremento de la testosterona. Este incremento de los niveles de testosterona pudo 


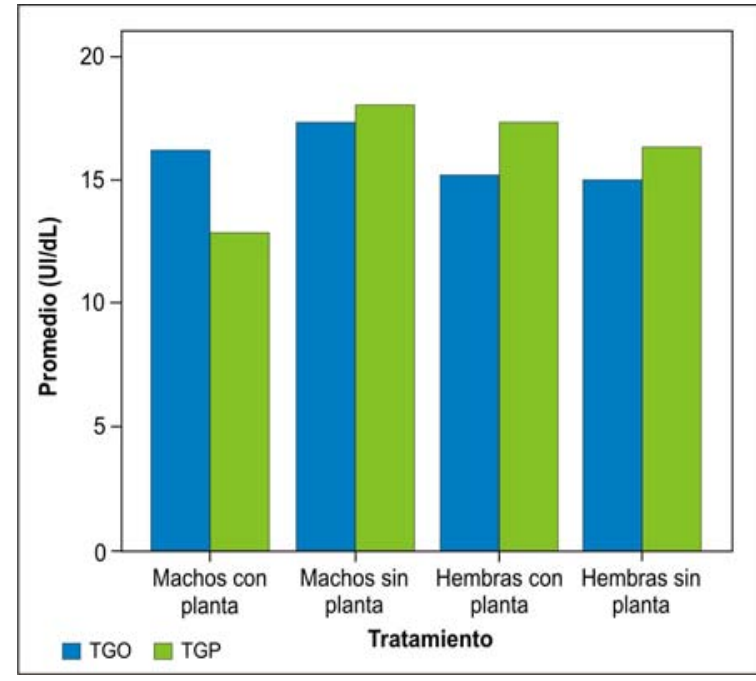

Figura 4. Nivel de transaminasas en suero de ratas después de 15 días de tratamiento.

haber inducido mayor número de copulaciones, sumándose al incremento de los niveles de FSH, que favorece la espermatogénesis y prepararía al endometrio para una mejor nidación del huevo fecundado. Esto se evidencia al observar gravidez en todas las ratas hembras expuestas a las ratas machos que recibieron la planta, comparativamente a las ratas hembras expuestas a las ratas machos que no recibieron planta (Figuras 6 y 7).

Es posible que los aminoácidos y esteroides presentes en el extracto etanólico de las flores de Laccopetalum giganteum (Tabla 1) contribuyan en mejorar la fertilidad $\left({ }^{13}\right)$. El sistema endocrino regula la producción de diversos tipos de hormonas, que se encuentran conformadas básicamente por aminoácidos y esteroles. Varios aminoácidos son requeridos por el organismo para la fabricación de neurotransmisores, tales como la dopamina y noradrenalina, que transmiten señales en el sistema del despertar sexual y el desempeño físico durante la actividad sexual. Los principales aminoácidos que estos neurotransmisores requieren incluyen fenilalanina, tirosina e histidina.

Además, los esteroles, saponinas, taninos y alcaloides actúan como una importante fuente energética y estructural. Previenen el daño espermatogénico e incrementan los recuentos espermáticos epididimarios.

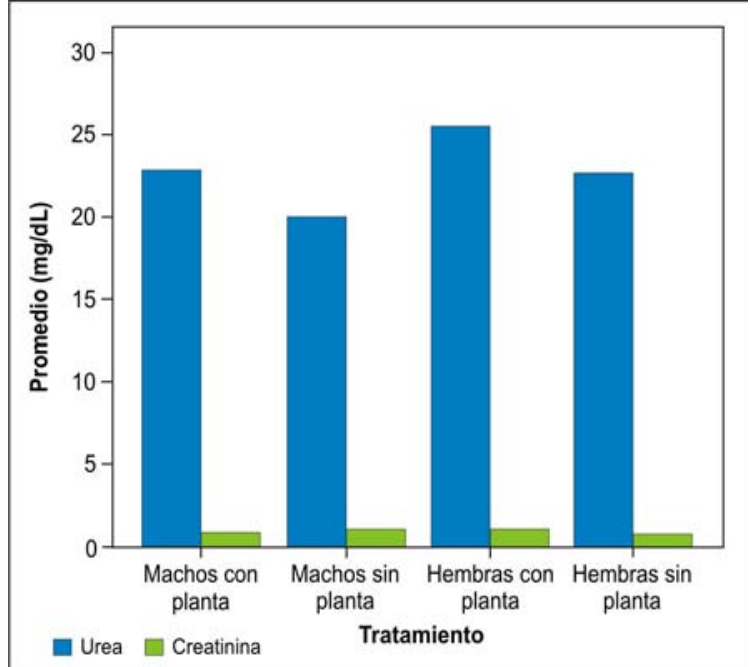

Figura 5. Nivel de urea y creatinina en suero de ratas después de 15 días de tratamiento.

Se ha demostrado que la arginina juega un rol muy importante en la fertilidad masculina, estimulando la producción y la motilidad de los espermatozoides. Se piensa que este aminoácido participa en la generación de óxido nítrico, el que a su vez viene siendo usado desde hace tiempo para contrarrestar la impotencia masculina $\left({ }^{14}\right)$. La histidina también se encuentra en altas concentraciones en algunas plantas, y se ha demostrado que desempeña una importante función en la eyaculación y el orgasmo. El organismo de los mamíferos utiliza la histidina para producir histamina y esta actúa sobre los cuerpos cavernosos del pene, los que a su vez participan activamente en el proceso de erección,

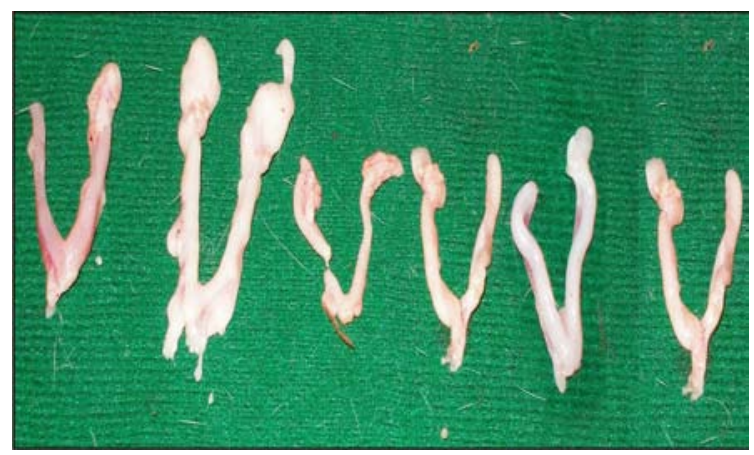

Figura 6. Gravidez de grupo control con suero fisiológico (machos y hembras juntos). 


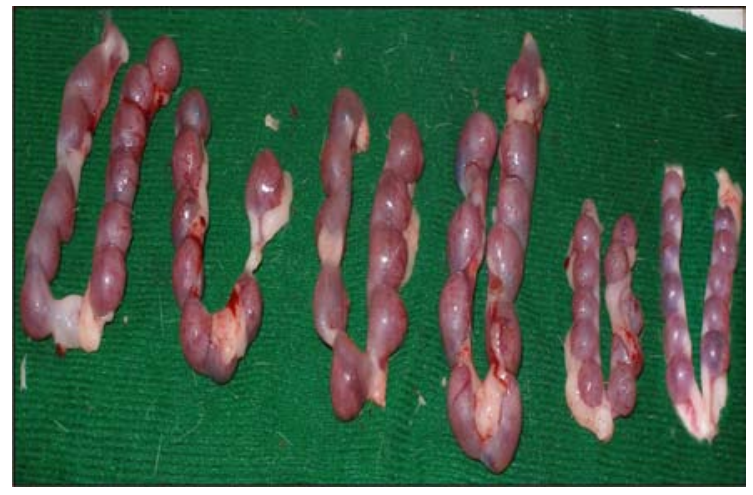

Figura 7. Gravidez de grupo tratamiento con pacra pacra (machos y hembras juntos).

desencadenando los estímulos que conducirán a la posterior eyaculación, lo que explicaría el aumento en el número de las cópulas. Además, algunos esteroles inhibirían la apoptosis $\left({ }^{15}\right)$ y pudiera ser éste el mecanismo que incrementa la celularidad de la espermatogénesis.

El estudio fitoquímico de las flores de Laccopetalum giganteum (pacra-pacra) ha evidenciado regular cantidad de aminoácidos (Tabla 1), por lo que se hace necesario mayores investigaciones al respecto, para conocer si la histidina y arginina estarían presentes y así explicar los postulados anteriores.

La determinación de los niveles de GOT, GPT (Figura 4), urea y creatinina (Figura 5) han mostrado que la planta no estaría induciendo una disfunción hepática y renal en las ratas a la dosis y tiempo ensayado, lo cual se trataría de un producto natural aceptable, pero que requiere de mayores estudios toxicológicos.

En conclusión, en condiciones experimentales, el extracto etanólico de las flores de Laccopetalum giganteum (pacra-pacra) evidenció efecto estimulante de la actividad reproductiva en ratas normales.

\section{REFERENCIAS BIBLIOGRÁFICAS}

1. Núñez R, García A, Cortés S, Sarrias O, Caballero P. Factores que influyen en los fallos de fecundación in vitro. Revista Iberoamericana de Fertilidad. 2001;18(5):130-6.
2. Palermo GD, Neri QV, Hariprashad JJ, Davis OK, Veeck LL, Rosenwaks Z. ICSI and its outcome. Semin Reprod Med. 2000;18(2):161-9.

3. European IVF-Monitoring Programme T.T. Assisted reproductive technology in Europe, 1997. Results generated from European registers by ESHRE. Hum Reprod. 2001;16(2):384-91.

4. Corns CM. Herbal remedies and clinical biochemistry. Ann Clin Biochem. 2003;40:489- 507.

5. Barnes J. Quality, efficacy and safety of complementary medicines: fashions, facts and the future. Part I. Regulation and quality. Br J Clin Pharmacol. 2003;55:226-33.

6. Rousseaux CG, Schachter H. Regulatory issues concerning the safety, efficacy and quality of herbal remedies. Birth Defects Res Part B. Dev Reprod Toxicol. 2003;68:505-10.

7. Gonzales GF, Gasco M, Córdova A, Cheng A. Effect of Lepidium meyenii (Maca), on spermatogenesis in male rats acutely exposed to high altitude $(4340 \mathrm{~m})$. J Endocrinol. 2004;180:87-95.

8. Zheng BL, He K, Kim CH, Rogers L, Shao Y, Huang ZY. Effect of lipidic extract from Lepidium meyenii on sexual behavior in mice and rats. Urology. 2000;55:598-602.

9. Lock de Ugaz O. Investigación fitoquímica. Métodos en el estudio de productos naturales. 2da ed. Lima: Fondo Editorial Pontificia Universidad Católica del Perú; 1994. p.1-7.

10. Cronquist A. The evolution and classification of flowering plants. New York: The New York Botanical Garden; 1988. p. 555.

11. Guyton A, Hall J. Tratado de Fisiología Médica. 9na Ed. Madrid: McGraw-Hill Interamericana; 1997. p. 1013-7.

12. Griswold MD, McLean D. Control of spermatogenesis via Sertoli cells. En: Rommerts FFG, Teerds KJ, eds. Testicular tangrams. Berlin: Springer; 2002. p. 75-84.

13. Piacente S, Carbone V, Plaza A, Zampelli A, Pizza C. Investigation of the tuber constituents of Maca (Lepidium meyenii Walp). J Agric Food Chem. 2002;50:5621-5.

14. Scibona M, Meschini P, Capparelli S, Pecori C, Rossi P, Menchi-Fabris GF. L-arginine and male infertility. Minerva Urol Nefrol. 1994;46:251-3.

15. Park TH, Kwon OS, Park SY, Han ES, Lee CS. N-methylated beta carbolines protect PC 12 cells from cytotoxic effect of MTP + by attenuation of mitochondrial membrane permeability change. Neurosci Res. 2003;46:349-58.

Manuscrito recibido el 02 de julio de 2007 y aceptado para publicación el 26 de setiembre de 2007.

Correspondencia:

Dr. Jorge Luis Arroyo Acevedo

Sección de Farmacología

Facultad de Medicina, UNMSM.

Av. Grau 750. Lima 1, Perú.

Correo-e: jorgeluis_arroyoacevedo@yahoo.es 